\title{
Combined Enhancement Algorithm Based on Muti-Scale Analysis
}

\author{
YUAN Wei-guo \\ Applied Technology School, \\ University of Science \& Technology Liaoning \\ Anshan, China \\ y189@163.com
}

\author{
WANG Song \\ Anshan longteng network co., LTD \\ Anshan, China \\ 18802183@qq.com
}

\begin{abstract}
Based on combinations of wavelet and curvelet transforms, an adaptive combined enhancement algorithm (ACEA) was proposed to solve the problems of the conventional method for image enhancement based on wavelet transform that the enhancement of edges whose contrast was same but angles were different was greatly different. The algorithm uses the wavelet transform to enhancement homogeneous areas and the curvelet transform to enhancement areas with edges. Simulation experiment results demonstrated that the algorithm got better cross entropy and CORR, improved effectively the effect of edge enhancement with good visual effect.
\end{abstract}

Keywords- wavelet transform; curvelet transform; muti-scale analysis

\section{INTRODUCTION}

Image enhancement is an important part of image processing field. Due to various external factors, such as light, sensor resolution, so that the original image has different level fuzzy edge, local or overall contrast and other deficiencies. Therefore enhancement algorithm should have the edge enhancement, improved contrast and other functions, and can effectively suppress noise ${ }^{[1]}$. In recent years, the theory of wavelet analysis is subject to a number of subjects of common concern. Wavelet transform is the inheritance and development of traditional Fourier transform. The multi-resolution analysis of wavelet has good space domain and frequency domain localization characteristic, for high frequency use gradually fine time or space step, focuses the analysis object of any details, thus it is especially suitable for the image signal of this class of non-stationary source processing, and it has become a signal / image processing methods ${ }^{2,3]}$. Curvelet transform is the second wavelet transform, can be more suitable for image processing features of a multi-scale transform, it can also have access to image in the smooth area and edge portions of the sparse representation, and has good directivity, some preliminary results in image processing of development potential ${ }^{[4,5]}$. Velde proposed weak edge enhancement algorithm which based on wavelet transform ${ }^{[6]}$. First, binary wavelet image decomposition, the wavelet coefficients are corrected in order to achieve the purpose, edge enhancement. But based on the wavelet transform contrast enhancement method for anisotropic feature detection is not adaptive, so the nonlinear edge enhancement will be able to achieve the ideal effect, While the Curvelet transformation can play a very good effect to various shaped edge enhancement [due to the highly directional sensitivity element ${ }^{[7,8]}$. According to two methods of enhancing their respective advantages, the study proposed the ACEA algorithm to enhance image processing.

\section{COMBINED ENHANCEMENT ALGORITHM}

\section{A. DWT Enhancement Method}

Compared with the Fourier transform, wavelet transform has airspace (time domain) and frequency domain" zoom" analysis ability. On the function $f(m, n)$ of twodimensional discrete wavelet transform, we can get a low pass sub-band $S_{j}$ and having a three orientation selective high-pass sub-band image $H_{i, j}$, continue the decomposition for the low pass band $S_{j}$ we can get the multi-level wavelet decomposition of $f(m, n){ }^{[9]}$. The decomposition results can be expressed as:

$$
\begin{aligned}
& W\{f(m, n)\}=\left\{\left(H_{i, j}=W_{i, j}[f(m, n)], S_{J}\right\}\right. \\
& 1 \leq i \leq 3,1 \leq j \leq J
\end{aligned}
$$

Type of $H_{i, j}-\mathrm{J}$ scale and direction of I details; $S_{J}$ - at the highest scale $\mathrm{J}$ image profile description, represents the image frequency information. The image frequency content focused on the low scale of high $H_{i, j}$, and the noise in the image part mainly focused on low scale $H_{i, j}$. In order to overcome the common enhancement method for noise often excessively enhanced disadvantages, should be of different scale decomposition coefficients are enhanced in different degrees.

\section{B. Curvelet Enhancement Method}

The discrete Curvelet transform processes were shown in Fig.1, from the chart we can see that the curvelet transform can be divided into the following 4 steps:

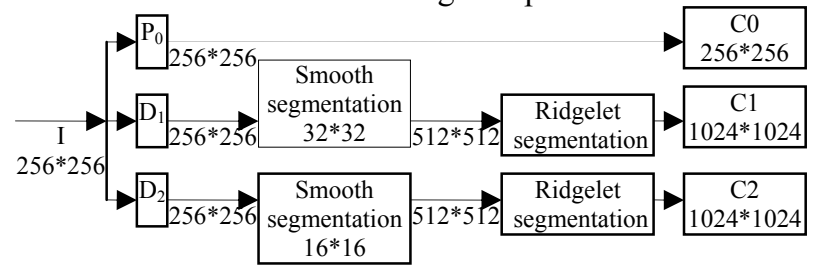

Figure 1. Curvelet transform processes

1) Subband decomposition 
In the decomposition process of Curvelet transform, to the first subband decomposition, we use filter $p_{0}, D_{s}$

$(s \geq 0$ ) decompose $I$ as:

$$
I \mid \rightarrow\left(p_{0} I, D_{1} I, D_{2} I \ldots \ldots\right)
$$

\section{2) Smooth segmentation}

In order to realize smooth segmentation, define the smoothing window $w_{Q}\left(x_{1}, x_{2}\right)$, the window is located on the square area:

$$
Q=\left[\frac{k_{1}}{2^{s}}, \frac{k_{1}+1}{2^{s}}\right) \times\left[\frac{k_{2}}{2^{s}}, \frac{k_{2}+1}{2^{s}}\right)
$$

The window with the function multiplication is located in the $Q$ square area. The segmentation process is applied to each sub-band $I$ :

$$
D_{s} I \mid \rightarrow\left(w_{Q} D_{s} I\right)_{Q \in Q_{s}}
$$

\section{3) Renormalization}

On each box $Q$, definition:

$$
\left(T_{Q} I\right)\left(x_{1}, x_{2}\right)=2^{s} I\left(2^{s} x_{1}-k_{1}, 2^{s} x_{2}-k_{2}\right)
$$

On the smooth blocks obtained after renormalization:

$$
g_{Q}=\left(T_{Q}\right)^{-1}\left(w_{Q} D_{s} I\right), Q \in Q_{s}
$$

\section{4) Ridgelet decomposition}

Finally, the base function for $\rho_{\lambda}$ orthogonal ridgelet decomposition, the decomposition process is shown in Fig. 2. First the images Radon transform, and then perform a one-dimensional wavelet transform. Because Curvelet transformation uses a highly directional sensitivity element, so in a variety of different shapes of edge enhancement it can play a very good effect. In Curvelet enhancement algorithm, the definition of a function $y_{c}$ on Curvelet transform coefficients are corrected.

$$
\begin{aligned}
& y_{c}(x, \sigma)=1, \text { if }(x<c \sigma) \\
& y_{c}(x, \sigma)=\frac{x-c \sigma}{c \sigma}\left(\frac{m}{c \sigma}\right)^{p}+\frac{2 c \sigma-x}{c \sigma}, \text { if }(x<2 c \sigma) \\
& y_{c}(x, \sigma)=\left(\frac{m}{x}\right)^{p}, \text { if }(2 c \sigma \leq x<m) \\
& y_{c}(x, \sigma)=\left(\frac{m}{x}\right)^{s}, \text { if }(x \geq m)
\end{aligned}
$$

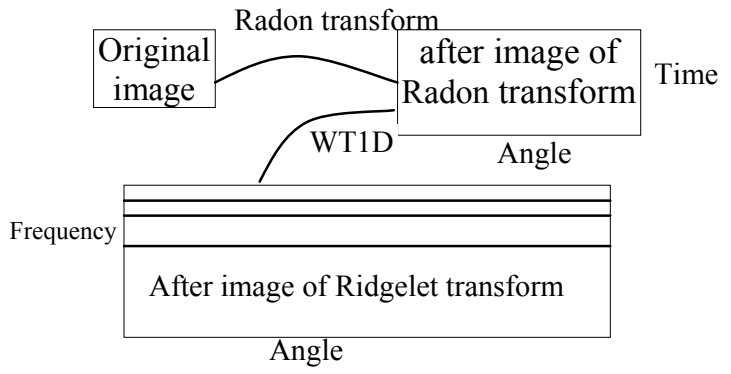

Figure 2. Ridgelet transform processes.
Let $m=c=p=0.5, s=0, p$ decided to nonlinearity, said $s$ image saturation; $c$ as the normalized parameter, when $c>3$ ensure that noise will not be enhanced; $m$ can be determined by the noise standard deviation $m=k_{m} \sigma$ or from a Curvelet transform maximum coefficient $m=L M c, L<1^{[11,12]}$. The image contrast enhancement process based on the Curvelet transform is as follows:

- Estimated input image standard noise variance A.

- Calculation of image Curvelet coefficient, $w_{j}$ are a group of subbands, each sub-band $w_{j}$ contains coefficients $N_{j}$, corresponding to a given resolution.

- A Curvelet transform is calculated for each subband $w_{j}$ standard noise variance.

- For every $w_{j}$ calculations in which the maximum value is $M_{j}$, then $y\left(\left|w_{j, k}\right|, \sigma_{j}\right)$ and Curvelet transform coefficients multiplication.

- The revised Curvelet coefficient reconstruction image, has realized the image contrast enhancement.

\section{The CEA algorithm}

The algorithm consists of the following four steps:

1) Divide image I into a smooth area and edge portion. The smooth area can be calculated through the image to get the squares, each pixel is defined as:

$$
V[i, j]=\sum_{p, q \in c}(I(i, j)-I(p, q))^{2}
$$

Here $C$ is $11 \times 11$ window centered at $[i, j]$, for the $I$ of an image smoothing, image square difference should be close to the noise variance of $\sigma^{2}$, can be through the threshold to detect the smoothed pixel:

$$
V[i, j] / \sigma^{2} \leq(1+\delta)
$$

$\delta$ is a very small number, other do not meet the conditions of the pixel is defined as an edge pixel.

2) Do enhanced DWT to image I in smooth regions.

3) Do enhanced Curvelet to image I in the edge portion.

4) Use B and C two obtained images fusion. Set smooth region pixel value is $I_{1}(x, y)$, the edge region of the pixel value of $I_{2}(x, y)$,After calculates the new pixel nephew of $J(x, y)$, where $x, y \in N$. A spatial point process, each pixel operations. In order to ease of handling, the two image is divided into $3 \times 3$ rectangular region, in the process, setting a fusion function, satisfy the following relations: $J(x, y)=F \times S(x, y)\left[I_{1}(x, y)-I_{2}(x, y)\right]+P \times I_{2}(x, y)$

Among them, $F$ fusion factor, provides the algorithm step, here $F=0.1, S(x, y)$ is a with the position 
coordinates of related coefficient, decision fusion is the image gradient method. $S(x, y)$ is:

$$
S(x, y)=\sqrt{\left(x-R_{x}\right)^{2}+\left(y-R_{y}\right)^{2}}
$$

Where $R_{x}, R_{y}$ is a rectangular area of the center.

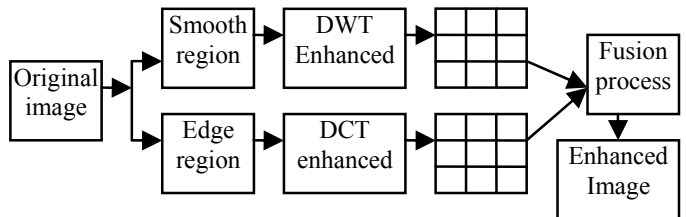

Figure 3. CEA principle diagram.

\section{THE EXPERIMENTAL RESULTS AND EVALUATION}

\section{A. Cross entropy}

The study can enhance the results of the evaluation of the image fusion statistical parameters including the image of the cross entropy, and contrast image correlation coefficient.

Cross entropy can be used to measure the two image gray distribution information. The smaller cross entropy is, the smaller the difference between the fused image and the reference image, the better. If a standard reference image is the $\mathrm{R}$, the fusion image is $\mathrm{F}$, then the cross entropy:

$$
C E=\sum_{i=0}^{L-1} P_{R_{i}} \log \frac{P_{R_{i}}}{p_{F_{i}}}
$$

Among them, $P_{R_{i}}$ is the image R gray value of I pixels and image pixels total ratio, and $P_{F_{i}}$ is the image $\mathrm{F}$ gray value of I pixels and image pixels total ratio.

\section{B. Correlation coefficient CORR}

Image correlation coefficient reflects certain relation of the two images.

$$
\operatorname{CORR}=\frac{\sum_{i=1}^{M} \sum_{j=1}^{N}[R(i, j)-\bar{R}][F(i, j)-\bar{F}]}{\sqrt{\left(\sum_{i=1}^{M} \sum_{j=1}^{N}[R(i, j)-\bar{R}]\right)^{2}\left(\sum_{i=1}^{M} \sum_{j=1}^{N}[F(i, j)-\bar{F}]\right)^{2}}}
$$

Among them, $\bar{R}, \bar{F}$ respectively for image $R, F$ average gray value. The experimental results are shown in table 1 .

TABLE I. IMAGE ENHANCEMENT EVALUATION INDEX

\begin{tabular}{c|c|c}
\hline Evaluation index & Cross entropy & $\begin{array}{c}\text { Correlation } \\
\text { coefficient }\end{array}$ \\
\hline $\begin{array}{c}\text { Wavelet } \\
\text { enhancement } \\
\text { algorithm }\end{array}$ & 2.168 & 0.9624 \\
\hline ACEA algorithm & 2.017 & 0.9738 \\
\hline
\end{tabular}

On the Lena image processing, such as Fig.4, respectively, using the traditional wavelet enhancement algorithm and CEA algorithm, the algorithm processing results was shown in Fig.5; the new algorithm processing results as shown in fig. 6 .

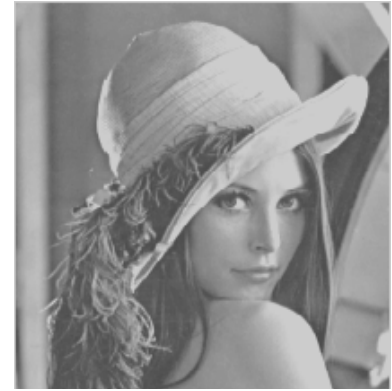

Figure 4. Original image.

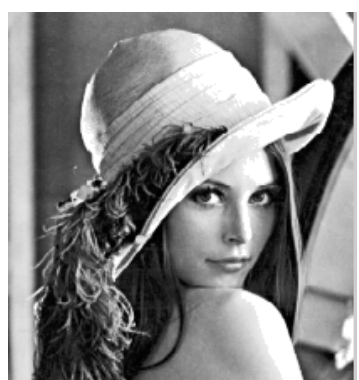

Figure 6. Algorithm of CEA image and histogram.

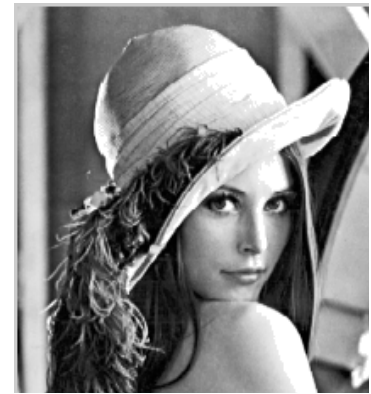

Figure 5.Wavelet enhancement processing to the image and histogram.

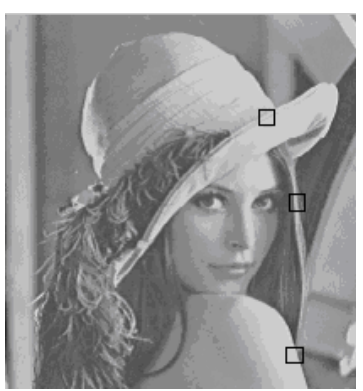

Figure 7. Calculation of CII range.
Edge enhancement effect uses a contrast improvement index CII (the contrast improvement index). CII is defined as:

$$
C I I=C_{\text {processed }} / C_{\text {original }}
$$

The image is divided into $3 \times 3$ small images, $c$ for all $3 \times 3$ scrap average value of image contrast, contrast is defined as $(\max -\min ) /(\max +\min )(\max$ is the maximum value of image gray value, while $\mathrm{min}$ is the minimum) - Calculation of Fig.7 demonstrates the improved contrast index; the results were shown in Table 2.

TABLE II. CONTRAST IMPROVEMENT INDEX

\begin{tabular}{c|ccc}
\hline $\begin{array}{c}\text { Contrast improvement } \\
\text { index }\end{array}$ & Brim rim & Arm edge & Hair edge \\
\hline $\begin{array}{c}\text { Wavelet enhancement } \\
\text { algorithm }\end{array}$ & 1.4640 & 1.7853 & 1.9724 \\
\hline ACEA algorithm & 1.8921 & 2.0974 & 2.7592 \\
\hline
\end{tabular}

\section{CONLUSION}

The study presents an enhanced algorithm, which combines wavelet transform multi-scale analysis capabilities, and Curvelet transform to direction of high sensitivity to image segmentation, the coefficients at different scales are enhanced in different degrees, to avoid a single means of 
enhancement method in image quality deficiencies. The test results show that, compared with traditional wavelet algorithm ACEA algorithm, great improvement of the contrast improvement index and enhancement of the effect is very good.

\section{ACKNOWLEDGMENT}

Conversations with Professor X.D.ZHANG and workmate C.Y. WHEN have been most helpful. This work has been supported by applied technology school.

\section{REFERENCES}

[1] Rafael C,Gonzalez, Richard E.Woods. Digital Image Processing[M].Second Edition. Englewood Cliffs, NJ: Prentice Hall, 2002,69-78.

[2] F. S. YANG. Wavelet transform and application of engineering analysis [M].Science Press,2003,132-153.
[3] BROWN TJ. An adaptive strategy for wavelet based image enhancement [A ]. Proceeding of Irish Machine Vision and Image Proceeding Conference[C ]. Belfast: Northern Ireland, 2000,256-275.

[4] W.F.CHEN. Wavelet analysis and its application in image processing [M]. Science Press,2003,112-123.

[5] Stephane Mallat,L.H.YANG. Wavelet signal processing to guide [M].Machine Press, 2002,167-187.

[6] X.ZHOU,S.D.ZHOU,F.HUANG,X.T.ZHOU. Image enhancement based on wavelet transform algorithm[J].Computer Applications, 2005,25(3):606-608.

[7] Machuca R, Phillips K. Application of vector fields to image processing[J]. IEEE Trans PAMI, 2006,15(5):316-329.

[8] Pitas I, Venetsanopoulos A N. Nonlinear digital filters principles and applications[M]. New York: Kluwer Academic Publishers, 1990.

[9] Astola J, Haavisto P, Neuvo Y. Vector median filter[C]. Proc. of IEEE Int Conf. on Image Processing, 2000, 78(2): 678-689.

[10] Trahanias P E, Venetsanopoulos A N. Vector directional filters proposed class of multichannel image processing filters[J]. IEEE Trans of Image Processing, 2003,23(2): 528-534. 